Pacific Journal of Mathematics

CIRCLE ACTIONS ON HOMOTOPY SPHERES WITH
CODIMENSION 4 FIXED POINT SET 


\title{
CIRCLE ACTIONS ON HOMOTOPY SPHERES WITH CODIMENSION 4 FIXED POINT SET
}

\author{
Ronald Fintushel and Peter Sie Pao
}

\begin{abstract}
In this paper we give a complete equivariant classification of smooth $S^{\prime}$ actions on homotopy spheres with codimension 4 fixed point set and point out a relationship with a natural generalization of the twist-spinning process for knots.
\end{abstract}

Semifree $S^{1}$ actions on homotopy spheres with codimension 4 fixed point set have been classified by J. Levine; so we concentrate on actions with exceptional orbits. There are some obvious linear models for these actions. Let $\xi$ be the standard generator of the complex representation ring of $S^{1}$. Then in some sense the actions with one exceptional orbit type are modeled after $\xi^{k} \oplus \xi \oplus 0$ and those with two exceptional orbit types are modeled after $\xi^{k} \oplus \xi^{m} \oplus 0$. Let $\Im_{k}^{n}$ denote the set of diffeomorphism classes of pairs $\left(\Sigma_{k}^{n-1}, \Delta\right)$ where $\Sigma^{n-1}$ is a homotopy $(n-1)$-sphere and $\Delta_{k}$ is a smooth $Z_{k}$-acyclic orientable codimension 2 submanifold with boundary an integral homology sphere. Similarly, for relatively prime integers $k$ and $m$, let $\S_{k, m}^{n}$ denote the set of triads $\left(\Sigma^{n-1} ; \Delta_{k}, \Delta_{m}\right)$ where $\Delta_{k}$ and $\Delta_{m}$ are respectively $Z_{k}$ and $Z_{m}$-acyclic orientable codimension 2 smooth submanifolds meeting tangentially such that $\partial \Delta_{k}=\partial \Delta_{m}=\Delta_{k} \cap$ $\Delta_{m}$ is an integral homology sphere. In these two cases the classification theorem states that actions on homotopy $n$-spheres with one exceptional orbit type $Z_{k}$, or two exceptional orbit types $Z_{k}$ and $Z_{m}$ are in 1-1 correspondence with $\Im_{k}^{n}$ and $\Im_{k, m}^{n}$. These 1-1 correspondences are realized by associating with an $S^{1}$ action on a homotopy $n$-sphere its structured orbit space and viewing $\Delta_{k}$ and $\Delta_{m}$ as the images in the orbit space of the fixed point sets of $Z_{k}$ and $Z_{m}$.

That these two types of actions do not comprise all $S^{1}$ actions on (homotopy) spheres with codimension 4 fixed point set was shown by $\mathrm{E}$. V. Stein in answer to a question of Frank Raymond. It turns out that all these other actions correspond in a 1-1 fashion via their structured orbit spaces to the set $\mathcal{T}_{k, m}^{n}$ of diffeomorphism classes of triads $\left(\Sigma^{n-1}, \Delta_{k}, \Delta_{m}\right)$ as in the definition of $\mathcal{S}_{k, m}^{n}$, except that the interiors of $\Delta_{k}$ and $\Delta_{m}$ intersect transversely in a (perhaps disconnected) $n-5$ manifold without boundary. This intersection manifold corresponds to the image in the orbit space of the exceptional orbits of type $Z_{k m}$. The intersecting aspect 
of these examples is that they have more orbit types than their slice representation at the fixed point set. Our description gives rise to an easy construction of all these actions.

Our classification of $S^{1}$ actions is carried out in terms of weak equivalence. (Recall that a weak equivalence of smooth $S^{1}$-manifolds $M_{1}$ and $M_{2}$ is a diffeomorphism $f: M_{1} \rightarrow M_{2}$ satisfying $f(t x)=a(t) f(x)$ for $t \in S^{1}, x \in M_{1}$ where $a$ is an automorphism of $S^{1}$.) Since $t \rightarrow t^{-1}$ is the only nontrivial automorphism of $S^{1}$, a weak equivalence of $S^{1}$-manifolds is just an equivariant diffeomorphism up to a change of direction of the action in one of the manifolds. This can be avoided by carrying along a specified orientation on the normal bundle in $\Sigma^{n-1}$ of $\partial \Delta_{k}$ (the fixed point set image), see [L]; however, we have opted for the simplicity that goes along with classification up to weak equivalence.

In $\$ 4$ we use $S^{1}$ actions on homotopy spheres to introduce a class of knots which generalize the twist-spin knots of Zeeman $[\mathbf{Z}]$. It is then a corollary of the classification theorem for $S^{1}$ actions that the knot complements of knots in this class fiber over the circle with fiber a punctured cyclic branched cover of the original knot. A special case of this is Zeeman's main theorem [Z]. Furthermore, this class of knots gives rise to infinitely many counterexamples to the $n$-dimensional Smith Conjecture.

The question of which homotopy spheres admit $S^{1}$ actions with codimension 4 fixed point set has been answered separately by J. Levine and R. Schultz in the semifree case. Recently Schultz has shown that any homotopy sphere which admits an $S^{1}$ action with codimension 4 fixed point set must also admit one which is semifree, and in fact he gives much more precise information $[\mathbf{S}]$. We wish to thank Reinhard Schultz for explaining his results to us and for encouraging the publication of our results. We also wish to thank Allan Edmonds for his excellent advice which has led to the restructing of our original format.

1. Orbit space and orbit structure. In this section we describe the general features of the orbit structure of a smooth $S^{1}$-action on a homotopy $n$-sphere $M^{n}$ whose fixed point set has codimension 4 . We identify the circle group $S^{1}$ with the group of complex numbers of unit modulus. If $S^{1}$ acts on $M$ and $X$ is a subset of $M$ then $X^{*}$ denotes its image in the orbit space $M^{*}$ and $p: M \rightarrow M^{*}$ denotes the orbit map. Given a subset $Y^{*}$ of $M^{*}$ we let $Y=p^{-1}\left(Y^{*}\right)$ when this causes no confusion. Let $F$ denote the fixed point set of $M$ and $E$ the union of the exceptional orbits (those with finite nontrivial isotropy group). The union of the exceptional orbits of type $Z_{k}$ will be called $E_{h}$, and $F_{h}$ will denote the fixed point set of $Z_{h}$ (so $\left.E_{h} \subset F_{h}\right)$. 
Let $S^{1}$ act smoothly on a homotopy $n$-sphere $M^{n}(n \geq 5)$, so $F$ is an integral homology sphere [B]. If $F$ is codimension 4 then the $S^{1}$-action on a slice $D^{n}$ at a point of $F$ must be equivalent to

$$
\begin{gathered}
S^{1} \times D^{2} \times D^{2} \times D^{n-4} \rightarrow D^{2} \times D^{2} \times D^{n-4}, \\
t \times\left(z_{1}, z_{2}, w\right) \rightarrow\left(z_{1} t^{k}, z_{2} t^{m}, w\right),
\end{gathered}
$$

where $k$ and $m$ are relatively prime positive integers. Since $F$ is connected this slice representation is the same at each point of $F$.

Proposition 1.1. If $k \geq 2$ (resp. $m \geq 2$ ) then $F_{k}$ (resp. $F_{m}$ ) is a smooth orientable $Z_{k}\left(\right.$ resp. $Z_{m}$ )-homology $n-2$ sphere.

Proof. Suppose $k \geq 2$. It is easily seen that $F_{k}$ is a smooth submanifold of $M^{n}$, and if $k$ is not a 2-power $F_{k}$ is orientable since it is the fixed point set of a $Z_{k}$-action ([B, IV.2.1]). For any prime $p$ dividing $k$ we have $F_{k} \subset F_{p}$; so $F_{p}$ is a $Z_{p}$-homology $n-2$ sphere. The connected component of $F_{h}$ which contains $F$ is a closed $n-2$ submanifold of $F_{p}$; hence $F_{h}=F_{p}$. It now follows from the universal coefficient theorem that $F_{k}$ is a $Z_{h}$-homology sphere. If $k$ is a 2-power it follows that $F_{k}$ is a $Z_{2}$-homology sphere and so is orientable.

Proposition 1.2. There are the following possibilities for the appearance of exceptional orbit types:

(i) no exceptional orbit types ( semifree action),

(ii) one exceptional orbit type,

(iii) $Z_{h}$ and $Z_{m}$-orbits,

(iv) $Z_{k}, Z_{m}$, and $Z_{k m}$-orbits, where in (iii) and (iv) $k$ and $m \geq 2$ and are relatively prime.

Proof. Let $t \times\left(z_{1}, z_{2}, w\right) \rightarrow\left(z_{1} t^{k}, z_{2} t^{m}, w\right)$ be the slice representation at a point of $F$. If $p$ is any prime then $F_{p^{s}}$ is a $Z_{p}$-homology sphere containing $F$. Thus if $k=m=1$ then (i) holds. If $k>1, m=1$ and $Z_{r}$ is an isotropy type, $r>1$, then each prime $p$ dividing $r$ also divides $k$. Let $p^{s}$ be the highest power of $p$ dividing $r$, then $F_{p^{s}}$ is a $Z_{p}$ homology $n-2$ sphere; so $F_{p}=F_{p^{s}}=F_{k}$. Suppose $r=p_{1}^{s_{1}} \cdots p_{u}^{s_{u}}$. Then $F_{r}=F_{p_{1}^{* 1}}$ $\cap \cdots \cap F_{p_{u}^{s u}}=F_{k}$. So $r=k$ since $Z_{r}$ and $Z_{k}$ are isotropy types.

If $k>1$ and $m>1$ and if $Z_{r}$ is an isotropy type with $r=p_{1}^{s_{1}} \cdots p_{u}^{s_{u}}>$ 1 , the above argument shows that each $F_{p_{i}^{s}}=F_{k}$ or $F_{m}$. Thus $F_{r}=F_{h}, F_{m}$, or $F_{k} \cap F_{m}=F_{k m}$. So $r=k, m$, or $k m$. 
Note that the submanifolds $F_{k}$ and $F_{m}$ meet transversely.

That $M^{*}$ is a topological manifold follows from slice considerations. We now describe how it can be smoothed. For details see the forthcoming paper of R. Schultz [S]. Consider first the semifree case which is presented in a paper of $\mathbf{J}$. Levine [L]. Let $N(F)$ be an equivariant tubular neighborhood of $F$ and note that $N^{*}\left(F^{*}\right)$ is the total space of a $D^{3}$-bundle over $F^{*}$. Since $p \mid F$ is a homeomorphism $F^{*}$ has an induced smooth structure and $N^{*}\left(F^{*}\right)$ thus inherits a smooth structure. Since the $S^{1}$-action on $M^{n}-F$ is free, $M^{*}-F^{*}$ also inherits a smooth structure. These structures agree on the overlap and so make $M^{*}$ a smooth manifold.

Consider next the case where the action has one exceptional orbit type $Z_{k}$. Then as above there is a smooth structure induced on $M / Z_{k}$ by the semifree $Z_{k}$-action and it can be seen that the induced semifree $S^{1} / Z_{k} \cong S^{1}$ action on $M / Z_{k}$ is smooth [S]. Hence $M^{*}=$ $\left(M / Z_{k}\right) /\left(S^{1} / Z_{k}\right)$ is a smooth manifold.

In case there are two or three exceptional orbit types we use the diagram:

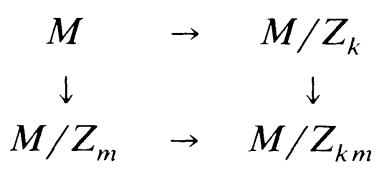

\section{$M^{*}$}

where each map is the orbit map of a semifree action. The diagram can be used in two different ways to make $M^{*}$ a smooth manifold; but it can be seen that the two smooth structures agree.

The existence of these smooth structures has been known for some time, and the following summary of their properties appears in $[\mathbf{S}]$.

Proposition 1.3. Let $M^{*}$ have the smooth manifold structure described above. Then:

(a) $p: M \rightarrow M^{*}$ is a smooth map.

(b) $p \mid F$ is a diffeomorphism and $F \rightarrow M^{*}$ is a smooth embedding.

(c) If $k>1(m>1)$ then $F_{k}^{*}\left(F_{m}^{*}\right)$ is smoothly embedded in $M^{*}$ extending the embedding of $F^{*}$.

(d) If the slice representation at $F$ is equivalent to

$$
\begin{gathered}
S^{1} \times \mathbf{C} \times \mathbf{C} \times R^{n-4} \rightarrow \mathbf{C} \times \mathbf{C} \times R^{n-4}, \\
t \times\left(z_{1}, z_{2}, w\right) \rightarrow\left(z_{1} t^{k}, z_{2} t^{m}, w\right)
\end{gathered}
$$


then:

(i) at $F, p$ is smoothly equivalent to

$$
\begin{gathered}
\mathbf{C} \times \mathbf{C} \times R^{n-4} \rightarrow \mathbf{C} \times R \times R^{n-4}, \\
\left(z_{1}, z_{2}, w\right) \rightarrow\left(z_{1}^{k} z_{2}^{-m},\left|z_{1}\right|^{2 k}-\left|z_{2}\right|^{2 m}, w\right) .
\end{gathered}
$$

(ii) at $E_{h}, p$ is smoothly equivalent to

$$
\begin{gathered}
\mathbf{C} \times R^{n-3} \times R \rightarrow \mathbf{C} \times R^{n-3}, \\
(z, w, s) \rightarrow\left(z^{k}, w\right),
\end{gathered}
$$

and a similar statement holds for $E_{m}$.

(iii) at $E_{k m}(k, m>1), p$ is smoothly equivalent to

$$
\begin{gathered}
\mathbf{C} \times \mathbf{C} \times R^{n-5} \times R \rightarrow \mathbf{C} \times \mathbf{C} \times R^{n-5}, \\
\left(z_{1}, z_{2}, w, s\right) \rightarrow\left(z_{1}^{k}, z_{2}^{m}, w\right) .
\end{gathered}
$$

Furthermore, any two smooth manifold structures $Q$ and $\mathscr{B}$ on $M^{*}$ which satisfy (a)-(d) are equivalent in the sense that there is a diffeomorphism $\left(M^{*}, \mathbb{Q}\right) \rightarrow\left(M^{*}, \Re\right)$ which is topologically isotopic to the identity.

Proposition 1.4. (a) $M^{*}$ is a homotopy $n-1$ sphere.

(b) $F_{k}^{*}, F_{m}^{*}, F_{k m}^{*}, F^{*}$ (and $F_{k}^{*} \cup F_{m}^{*}$ in case (iii) of Prop. 1.2) are orientable smooth submanifolds of $M^{*}$, and $\partial F_{k}^{*}=\partial F_{m}^{*}=F^{*}$.

(c) $F^{*}$ is an integral homology $S^{n-4} ; F_{k}^{*}\left(\right.$ resp. $\left.F_{m}^{*}\right)$ is $Z_{k}$-acyclic (resp. $Z_{m}$-acyclic). ( So in case (iii) of Prop. 1.2, $F_{k}^{*} \cup F_{m}^{*}$ is a rational homology $S^{n-3}$.)

(d) In case (iv) of Prop. $1.2 E_{k m}^{*}$ is an orientable closed $n-5$ submanifold of $M^{*}$ which is the transverse intersection of $E_{k}^{*}$ and $E_{m}^{*}$.

Proof. Part (a) follows from [CF]. Except for the orientability (b) follows from slice considerations and Prop. 1.3, and (c) follows from Smith theory and Prop. 1.1. To see that $F_{k}^{*}$ is orientable, let $\gamma^{*}$ be any loop in Int $F_{k}^{*}=F_{k}^{*}-F^{*}$. Since $E_{k m}^{*}$ is codimension 2 in $F_{k}^{*}$ an isotopy moves $\gamma^{*}$ into $E_{k}^{*}$. For a tubular neighborhood $N^{*}$ of $\gamma^{*}$ we have $p^{-1}\left(N^{*}\right)=N \cong N^{*} \times S^{1}$. But $N$ is orientable since $F_{k}$ is orientable, thus $N^{*}$ is orientable and $F_{k}^{*}$ is also. To prove (d) note that the $Z_{k m}$-action on a slice $D^{2} \times D^{2} \times D^{n-5}$ at a point of $E_{k m}$ must be

$$
e^{2 \pi l / k m} \times\left(z_{1}, z_{2}, w\right) \rightarrow\left(z_{1} e^{2 \pi l / m}, z_{2} e^{2 \pi i / k}, w\right) .
$$


All of (d) follows from this except for the statement about orientability. Since $k$ and $m$ are relatively prime, we may suppose that $m$ is odd. Now $E_{k m}$ is a component of the fixed point set of the induced $Z_{m}$ action on the orientable manifold $F_{k}$. Thus $E_{k m}$ is orientable ([B, IV.2.1]), and the argument given above to show $F_{k}^{*}$ is orientable also shows tht $E_{k m}^{*}$ is orientable.

2. Actions with one exceptional orbit type. Consider a smooth $S^{1}$ action on a homotopy $n$-sphere $M^{n}(n \geq 5)$ with codimension 4 fixed point set and one exceptional orbit type $Z_{k}$. Then $F_{k}^{*}$ is a smooth codimension 2 submanifold of $M^{*}$ with boundary $F^{*}$, where $M^{*}$ is a homotopy $n-1$ sphere, $F_{k}^{*}$ is an orientable $Z_{k}$-homology $n-3$ disk and $F^{*}$ is an integral homology $n-4$ sphere. Call the pair $\left(M_{k}^{*}, F^{*}\right)$ the structured orbit space of the action. In view of the above facts we define the set of potential structured orbit spaces $\delta_{k}^{n}$ to be the set of diffeomorphism classes of pairs $\left(\Sigma^{n-1}, \Delta_{k}\right)$, where $\Delta_{k}$ is a smooth orientable $n-3$ dimensional $Z_{k}$-acyclic submanifold of the homotopy $n-1$ sphere $\sum^{n-1}$ and $\partial \Delta_{k}$ is an integral homology $n-4$ sphere.

For a smooth $S^{1}$ action on a homotopy $n$-sphere $M^{n}(n \geq 5)$ with codimension 4 fixed point set and one exceptional orbit type $Z_{k}$, the structured orbit space $\left(M^{*}, F_{k}^{*}\right) \in \mathcal{S}_{k}^{n}$. We shall show in this section that for each $\sigma \in \mathcal{S}_{k}^{n}$ there is up to weak equivalence a unique action $M(\sigma)$ of $S^{1}$ on a homotopy $n$-sphere with structured orbit space $\sigma$.

Given $\sigma=\left(\sum^{n-1}, \Delta_{k}\right) \in \mathcal{S}_{k}^{n}$ we now proceed with the construction of $M(\sigma)$. According to $[\mathbf{L}]$ if we fix an orientation of the normal bundle of $\partial \Delta_{k}$ in $\Sigma^{n-1}$ we determine up to equivariant diffeomorphism a semifree $S^{1}$ action on a homotopy $n$-sphere $N^{n}$ with fixed point set $\bar{F} \cong \partial \Delta_{h}$ and smooth orbit map $\mu: N^{n} \rightarrow \Sigma^{n-1}$ with $\mu(\bar{F})=\partial \Delta_{h}$. Let $\bar{F}_{k}=\mu^{-1}\left(\Delta_{h}\right)$; so $\bar{F}_{k}$ is a smooth codimension 2 submanifold of $N^{n}$.

Claim. $\bar{F}_{k}$ is an orientable $Z_{k}$-homology sphere.

Proof. $\mu \mid \bar{F}_{k} \rightarrow \Delta_{k}$ is the orbit map of a semifree $S^{1}$ action with codimension 2 fixed point set. It follows from [B, V.10.1] that $\bar{F}_{k}$ is homeomorphic to $\Delta \times S^{1} /(x, t) \sim\left(x, t^{\prime}\right)$ for $t, t^{\prime} \in S^{1}$. I.e. $\bar{F}_{k}$ is homeomorphic to $\Delta_{k} \times S^{1} \cup \partial \Delta_{k} \times D^{2} \cong \partial\left(\Delta_{k} \times D^{2}\right)$; so $\bar{F}_{k}$ is orientable since $\Delta_{k}$ is orientable, and duality implies that $\bar{F}_{k}$ is a $Z_{k}$-homology sphere.

Since $H_{1}\left(N^{n}-\bar{F}_{k} ; Z_{k}\right) \approx H^{n-2}\left(\bar{F}_{k} ; Z_{k}\right) \approx Z_{k}$ there is a $k$-fold cyclic branched cover $M^{n}$ of $N^{n}$ branched over $\bar{F}_{k}$. According to [DK] $M^{n}$ has a unique smooth manifold structure such that $\nu: M^{n} \rightarrow N^{n}$ is the smooth 
orbit map of a smooth semifree $Z_{k}$ action, and such that the smooth structure on $N^{n}$ which is imposed via the semifree action (as in $\S 1$ ) is just the original smooth structure on $N^{n}$.

Claim. The $S^{1}$ action on $N^{n}$ lifts to a smooth $S^{1}$ action on $M^{n}$ containing the $Z_{k}$ action.

Proof. By [B, I.9] there is a unique $S^{1}$ action on $M-\nu^{-1}\left(\bar{F}_{h}\right)$ covering the $S^{1}$ action on $N-\bar{F}_{k}$; i.e. $\nu(t \cdot x)=t^{k} \cdot \nu(x)$ for all $x \in M-\nu^{-1}\left(\bar{F}_{k}\right)$ and $t \in S^{1}$. This action is smooth because the smooth structure of $M-\nu^{-1}\left(\bar{F}_{k}\right)$ is lifted from $N-\bar{F}_{k}$ via the cover. The normal bundle of $\bar{F}_{k}$ in $N^{n}$ is trivial [MS, $\left.\S 11\right]$; thus it follows from [B, VI.11.1] that the $S^{1}$ action on an equivariant normal bundle of $\bar{F}_{k}$ in $N$ is equivalent to

$$
\begin{gathered}
S^{1} \times \bar{F}_{k} \times D^{2} \rightarrow \bar{F}_{k} \times D^{2}, \\
t \times(x, z) \rightarrow\left(t \cdot x, z t^{u}\right)
\end{gathered}
$$

for some integer $u$, where $t \cdot x$ denotes the action of $S^{1}$ on $\bar{F}_{k}$. Now $M$ is diffeomorphic to the union of $M-\nu^{-1}\left(\bar{F}_{h}\right)$ with the tubular neighborhood $T$ of $\nu^{-1}\left(\bar{F}_{k}\right)$ in $M$, and $T$ can be identified with $\bar{F}_{k} \times D^{2}$ (see [DK; p. 160]). Under this identification $\nu \mid \bar{F}_{k} \times D^{2}$ is $\nu(x, z)=\left(x, z^{k}\right)$. Define the $S^{1}$ action on $T \cong \bar{F}_{k} \times D^{2}$ by $t \times(x, z) \rightarrow\left(t^{h} \cdot x, z t^{u}\right)$. This covers the $S^{1}$ action on $\bar{F}_{h} \times D^{2} \subset N$; so by uniqueness of covering action on $T-\nu^{-1}\left(\bar{F}_{h}\right)$ the actions on $T$ and $M-\nu^{-1}\left(\bar{F}_{h}\right)$ patch together to give a smooth $S^{1}$ action on $M$ containing the given $Z_{k}$ action.

Claim. $\pi_{1}(M)=0$.

Proof. By Van Kampen's theorem $\pi_{1}(M)$ is the free product of $\pi_{1}\left(M-\nu^{-1}\left(\bar{F}_{h}\right)\right)$ and $\pi_{1}(T)$ amalgamated over $\pi_{1}\left(T-\nu^{-1}\left(\bar{F}_{h}\right)\right)$. Now $M-\nu^{-1}\left(\bar{F}_{k}\right)=(\mu \nu)^{-1}\left(\Sigma^{n-1}-\Delta_{k}\right)$. But $\Delta_{k}$ is codimension 2 in $\Sigma^{n-1}$ and $\partial \Delta_{h} \neq 0$; so the usual piping argument shows that $\pi_{1}\left(\sum^{n-1}-\Delta_{h}\right)=0$. Hence the exact sequence

$$
\pi_{1}\left(S^{1}\right) \rightarrow \pi_{1}\left((\mu \nu)^{-1}\left(\Sigma^{n-1}-\Delta_{h}\right)\right) \rightarrow \pi_{1}\left(\Sigma^{n-1}-\Delta_{k}\right)=0
$$

shows that $\pi_{1}\left(M-\nu^{-1}\left(\bar{F}_{h}\right)\right)$ is generated by a circle orbit. Since the $S^{1}$ action on $M$ has a nontrivial fixed point set $\pi_{1}\left(M-\nu^{-1}\left(\bar{F}_{h}\right)\right) \rightarrow \pi_{1}(M)$ is the 0 -map. Also, since $\partial T \rightarrow \nu^{-1}\left(\bar{F}_{k}\right)$ is an $S^{1}$ bundle projection, the homomorphism $\pi_{1}\left(T-\nu^{-1}\left(\bar{F}_{k}\right)\right)=\pi_{1}(\partial T) \rightarrow \pi_{1}\left(\nu^{-1}\left(\bar{F}_{k}\right)\right)=\pi_{1}(T)$ is onto. It follows that $\pi_{1}(M)=0$. 
Now for $R=Q$ or $Z_{p}, p$ prime not dividing $k$, we have

$$
H_{*}(M ; R) \approx H_{*}(M ; R)^{Z_{k}} \approx H_{*}(N ; R) \approx H_{*}\left(S^{n} ; R\right)
$$

where the first isomorphism is due to the fact that $Z_{k}$ action is contained in the $S^{1}$ action and the second isomorphism follows from [B, III.2.4] since $p$ is prime to $k$.

Claim. $H_{*}\left(M^{n} ; Z_{k}\right) \approx H_{*}\left(S^{n} ; Z_{k}\right)$.

Proof. Since $\Delta_{k}$ is $Z_{k}$ acyclic, Alexander duality implies that $\Sigma^{n-1}-\Delta_{k}$ is $Z_{k}$ acyclic. But $M-\nu^{-1}\left(\bar{F}_{k}\right)=(\mu \nu)^{-1}\left(\Sigma^{n-1}-\Delta_{k}\right)$ is a circle bundle over $\Sigma^{n-1}-\Delta_{k}$; hence $M-\nu^{-1}\left(\bar{F}_{k}\right)$ has the $Z_{k}$-homology of a circle. We have seen above that the tubular neighborhood $T$ of $\nu^{-1}\left(\bar{F}_{k}\right)=F_{k}$ is $T \cong F_{k} \times D^{2}$, and $F_{k} \cong \bar{F}_{k}$ is a $Z_{k}$ homology $n-2$ sphere. The claim now follows from a simple Mayer-Vietoris argument.

It now follows from the universal coefficient theorem that $H_{*}(M, Z)$ $\approx H_{*}\left(S^{n} ; Z\right)$; so $M$ is a homotopy $n$ sphere. Letting $M(\sigma)=M$ we have proved the realization theorem:

THEOREM 2.1. Given $\sigma \in \mathcal{S}_{k}^{n}$ there is a smooth $S^{1}$ action on a homotopy $n$-sphere $M(\sigma)$ with structured orbit space $\sigma$.

In order to prove that this action is unique we need the next lemma.

Lemma 2. Let $X$ and $Y$ be $S^{1}$ spaces and consider the actions of $Z_{k}$ embedded in the $S^{1}$ actions. Suppose we have the commutative diagram

$$
\begin{array}{ccc}
X & \stackrel{f}{\rightarrow} & Y \\
\pi, \downarrow & & \downarrow \pi_{v} \\
X / Z_{k} & \stackrel{g}{\rightarrow} & Y / Z_{k}
\end{array}
$$

where $f$ is $Z_{k}$-equivariant and $g$ is $S^{1}$-equivariant with respect to the induced actions. Then $f$ is $S^{1}$-equivariant.

Proof. Let $t \in S^{1}$ and $x \in X$. Then

$$
\begin{aligned}
\pi_{Y}(f(t \cdot x)) & =g\left(\pi_{X}(t \cdot x)\right)=g\left(t \cdot \pi_{X}(x)\right)=t \cdot g\left(\pi_{X}(x)\right) \\
& =t \cdot \pi_{Y}(f(x))=\pi_{Y}(t \cdot f(x)) .
\end{aligned}
$$


Thus there is an $s \in Z_{k}$ such that $t \cdot f(x)=s \cdot f(t \cdot x)$. But $s=1$ when $t=1$, so a continuity-connectedness argument implies that $s$ is always 1; so $f$ is $S^{1}$-equivariant.

THEOREM 2.3. Let $M_{1}^{n}$ be a smooth $S^{1}$-manifold with codimension 4 fixed point set and one exceptional orbit type $Z_{k}$. Suppose that $M_{1}^{n}$ has structured orbit space $\sigma \in \mathcal{S}_{k}^{n}$. Then $M_{1}^{n}$ is weakly equivalent to $M(\sigma)$; in particular $M_{1}^{n}$ is a homotopy sphere.

Proof. Let $f^{*}$ be a diffeomorphism of pairs $\left(M_{1}^{*}, F_{k}^{*}\left(M_{1}\right)\right) \rightarrow$ $\left(\Sigma^{n-1}, \Delta_{k}\right)=\sigma$. As in $\S 1, M_{1} / Z_{k}$ has an inherited smooth structure such that $M_{1}$ is the smooth $k$-fold cyclic branched cover of $M_{1} / Z_{k}$ branched over $\bar{F}_{k}\left(M_{1}\right)$. The induced $S^{1} / Z_{k}$ action on $M_{1} / Z_{k}$ is semifree and (after if necessary a change in the orientation of the $S^{1}$ action on $M_{1}$ ) Levine's theorem [L] yields an $S^{1}$-equivariant diffeomorphism $\bar{f}$

$$
\begin{array}{ccc}
M_{1} / Z_{k} & \stackrel{\bar{f}}{\rightarrow} & N \\
\mu_{1} \downarrow & & \downarrow \mu \\
M_{1}^{*} & \stackrel{f^{*}}{\rightarrow} & \sum^{n-1}
\end{array}
$$

Note that

$$
\bar{f}\left(\bar{F}_{k}\left(M_{1}\right)\right)=\mu^{-1} f^{*} \mu_{1}\left(\bar{F}_{k}\left(M_{1}\right)\right)=\mu^{-1} f^{*}\left(F_{k}^{*}\left(M_{1}\right)\right)=\mu^{-1}\left(\Delta_{k}\right)=\bar{F}_{k} .
$$

By the uniqueness of smooth cyclic branched covers [DK] there is a $Z_{k}$-equivariant diffeomorphism $f$ :

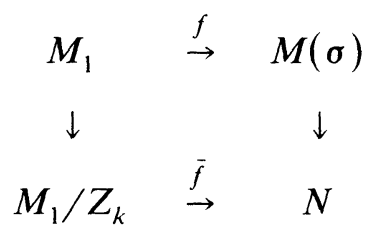

and $f$ is $S^{1}$-equivariant by Lemma 2.2 .

3. Actions with 2 or 3 exceptional orbit types. The classification theorem of the previous section is the main tool used in studying smooth $S^{1}$ actions on homotopy $n$-spheres with codimension 4 fixed point set and two or three exceptional orbit types. For $n \geq 5$ and $k, m$ relatively prime positive integers define $\mathcal{S}_{k, m}^{n}$ to be the set of diffeomorphism classes of triads $\left(\Sigma^{-1} ; \Delta_{k}, \Delta_{m}\right)$ where $\Sigma^{n-1}$ is a homotopy sphere, $\Delta_{k}$ is a smooth orientable $Z_{k}$-acyclic $n-3$ submanifold, $\Delta_{m}$ is a smooth orientable $Z_{m}$ 
acyclic $n-3$ submanifold, $\Delta_{h} \cap \Delta_{m}=\partial \Delta_{h}=\partial \Delta_{m}$ is an integral homology $n-4$ sphere, and $\Delta_{h} \cup \Delta_{m}$ is a smooth submanifold of $\sum^{n-1}$.

Define $\sigma_{k, m}^{n}$ to be the set of diffeomorphism classes of triads $\left(\Sigma^{n-1}, \Delta_{h}, \Delta_{m}\right)$ as above except that Int $\Delta_{h}$ and Int $\Delta_{m}$ meet transversely in $Q$ a (perhaps disconnected) $n-5$ dimensional smooth submanifold, and $\Delta_{h} \cup \Delta_{m}-Q$ is a smooth submanifold of $\Sigma^{n-1}$.

If $S^{1}$ acts smoothly on a homotopy $n$-sphere $M^{n}$ with codimension 4 fixed point set and two or three exceptional orbit types we call $\left(M^{*}\right.$; $\left.F_{h}^{*}, F_{m}^{*}\right)$ the structured orbit space of the action. If $n \geq 5$ it follows from $\S 1$ that $\left(M^{*} ; F_{h}^{*}, F_{m}^{*}\right) \in \mathcal{S}_{h, m}^{n}$ or $\widetilde{T}_{h, m}^{n}$.

THeOREM 3.1. Suppose that the $S^{1}$-manifolds $M_{1}$ and $M_{2}$ have the same structured orbit space $\sigma \in \mathcal{S}_{h, m}^{n}$ or $\sigma_{h, m}^{n}$. Then $M_{1}$ and $M_{2}$ are weakly equivalent.

Proof. Let $f^{*}$ be a diffeomorphism $\left(M_{1}^{*} ; F_{h}^{*}\left(M_{1}\right), F_{m}^{*}\left(M_{1}\right)\right) \rightarrow\left(M_{2}^{*}\right.$; $\left.F_{h}^{*}\left(M_{2}\right), F_{m}^{*}\left(M_{2}\right)\right)$. The induced $S^{1}$ action on $M_{i} / Z_{m}$ has one exceptional orbit type $Z_{h}$ and structured orbit space $\left(M_{l}^{*}, F_{h}^{*}\left(M_{l}\right)\right)$, so by Theorem 2.3 there is a weak equivalence $f: M_{1} / Z_{m} \rightarrow M_{2} / Z_{m}$ covering $f^{*}$. Hence $f\left(F_{m}\left(M_{1}\right) / Z_{m}\right)=F_{m}\left(M_{2}\right) / Z_{m}$. Now $M_{\imath}$ is the smooth $m$-fold cyclic branched cover of $M_{l} / Z_{m}$ branched over $F_{m}\left(M_{l}\right) / Z_{m}$; so we may apply the uniqueness of branched covers and Lemma 2.2 to finish the argument as in the proof of Theorem 2.3.

The rest of this section is devoted to showing that for each $\sigma \in \varsigma_{h, m}^{n}$ or $\sigma_{h, m}^{n}$ there is a smooth $S^{1}$-action $M(\sigma)$ on a homotopy $n$-sphere with structured orbit space $\sigma$. So let $\sigma=\left(\sum^{n-1} ; \Delta_{h}, \Delta_{m}\right) \in \mathcal{S}_{h, m}^{n}$ or $\sigma_{h, m}^{n}$. Proceeding as in $\S 2$ we may orient the normal bundle of $\partial \Delta_{h}=\partial \Delta_{m}$ in $\Sigma^{n-1}$ and obtain a smooth semifree $S^{1}$ action on a homotopy sphere $N^{n}$ with orbit map $\mu: N \rightarrow \Sigma^{n-1}$ and fixed point set $\mu^{-1}\left(\partial \Delta_{h}\right)$. Let $\bar{F}_{h}=$ $\mu^{-1}\left(\Delta_{h}\right)$ and $\bar{F}_{m}=\mu^{-1}\left(\Delta_{m}\right)$. As in $\S 2$ the respective $k$ and $m$-fold cyclic branched covers $M_{h}$ and $M_{m}$ are homotopy $n$-spheres with smooth $S^{1}$ actions and structured orbit spaces $\left(\sum^{n-1}, \Delta_{h}\right)$ and $\left(\sum^{n-1}, \Delta_{m}\right)$. Now form the pullback diagram:

$$
\begin{array}{ccc}
M & \stackrel{\nu_{m}^{\prime}}{\rightarrow} & M_{h} \\
\nu_{h}^{\prime} \downarrow & & \downarrow \nu_{h} \\
M_{m} & \stackrel{\nu_{m}}{\rightarrow} & N
\end{array}
$$


The pullback $M$ is a topological manifold because $\bar{F}_{k}$ and $\bar{F}_{m}$ meet transversely in $N$. Also $\nu_{k}^{\prime}$ and $\nu_{m}^{\prime}$ are branched $k$ and $m$-fold covering projections. So $M$ inherits a smooth structure as in $\$ 2$ in two different ways and the $S^{1}$-actions lift to $S^{1}$ actions on $M$, smooth in their respective structures. However since both actions have the same structured orbit space $\sigma$, by Theorem 3.1 they are weakly equivalent. In particular the above two smooth manifold structures on $M$ are diffeomorphic.

THEOREM 3.2. For each $\sigma \in \mathcal{S}_{h, m}^{n}$ or $\widetilde{T}_{h, m}^{n}$ there is a homotopy $n$-sphere $M(\sigma)$ with a smooth $S^{1}$ action with $\sigma$ as structured orbit space.

Proof. Let $M(\sigma)=M$ above. As in $\S 2$ for $R=Q$ or $Z_{p}$ for $p$ any prime not dividing both $k$ and $m$ (hence for any prime $p$ ) $H_{*}(M ; R)$ $\approx H_{*}\left(S^{n} ; R\right)$; thus $H_{*}(M, Z)=H_{*}\left(S^{n} ; Z\right)$. So to show that $M=M(\sigma)$ is a homotopy sphere we must show that $M$ is simply connected.

To this end consider a loop $\lambda$ in $M^{n}(n \geq 5)$ based at a point in a principal orbit, and by general position suppose $\lambda$ misses $E \cup F$. Let $p$ : $M \rightarrow \Sigma$ be the orbit map. The based loop $p \lambda$ can be homotoped in $\Sigma-E^{*} \cup F^{*}$ to an embedding, and this homotopy can be lifted to $M$. Hence we may suppose that $\lambda$ and $p \lambda$ are embedded loops. Since $\pi_{1} \Sigma=0$, $p \lambda$ bounds a transversely immersed 2-disk $D$ which meets $E^{*} \cup F^{*}$ transversely in a finite number of points and which misses $E_{k m}^{*}$. By sliding intersections of $D$ with $E^{*} \cup F^{*}$ to $F^{*}$ we may arrange that $D \cap\left(E^{*} \cup\right.$ $\left.F^{*}\right)=D \cap F^{*}$. If $D \cap F^{*}=\varnothing$ introduce an intersection point of $D$ and $F^{*}$ by pushing $D$ until it meets $F^{*}$ tangentially at a regular point of $D$. Now $p^{-1}(D) \cong D \times S^{1} / \sim$ where $\sim$ identifies $\{x\} \times S^{1}$ to $(x, 1)$ for each $x \in D \cap F^{*}$; so the map $p_{\sharp}: \pi_{1}(p \quad(D)) \rightarrow \pi_{1}(D)$ is an isomorphism, and $\lambda$ is nullhomotopic.

COROLlary 3.3. Let $M^{n}$ be a smooth $S^{1}$-manifold with structured orbit space $\sigma \in \mathbb{S}_{h, m}^{n}$ or $\widetilde{T}_{h, m}^{n}$. Then $M$ is a homotopy sphere.

In the introduction we mentioned a question posed by Frank Raymond [M; p. 353]. In our context it is equivalent to asking whether each $F_{q}$ is connected when $S^{1}$ acts on $S^{n}$. Our theory provides infinitely many counterexamples in each dimension $\geq 5$ for a given slice representation (1.3(d)) at the fixed point set determined by the integers $k$ and $m$. In fact we have described how all such examples with codimension 4 fixed point set can occur. As we have noted the first such example was constructed by Elliott Stein [St]. In his example the structured orbit space is $\left(S^{4}\right.$; $\left.D_{h}^{2}, D_{m}^{2}\right) \in \mathcal{T}_{h, m}^{5}$ where $D_{h}^{2} \cup D_{m}^{2}$ is the standard immersed $S^{2}$ in $S^{4}$ with one double point. 
Finally, we should mention that in the case $n=4$ analogous results hold. Essentially $\delta_{k}^{4}$ is the set of homotopy 3 -spheres and $\delta_{k, m}^{4}$ is the set of diffeomorphism classes of knots in homotopy 3-spheres; $\widetilde{T}_{h, m}^{4}=\varnothing$. See $[\mathbf{F}]$ and $[\mathbf{P}]$.

4. Twist-spinning knots. By a "knot" we shall mean a smooth pair $N^{n-3} \subset M^{n-1}$ of homotopy spheres. The $k$ twist-spin $(k>1)$ of the knot $N^{n-3} \subset M^{n-1}$ may be obtained as follows. Let $\left(D^{n-1}, D^{n-3}\right) \subset(M, N)$ be a standard disk pair and consider $\sigma=\left(M^{n-1}, D^{n-3}\right) \in \mathcal{S}_{k}^{n}$. We shall find it more convenient to use the notation $\sigma=\left(M^{n-1}, D^{n-3}, N^{n-3}-\right.$ Int $\left.D^{n-3}\right) \in \mathcal{S}_{k, 1}^{n}$. Write $F_{1}=p^{-1}\left(N^{n-3}-\right.$ Int $\left.D^{n-3}\right)$. By Theorem 2.3, $M(\sigma)$ is a homotopy sphere, and following the proof of the first claim of $\S 2$ we see that $F_{1}$ is also a homotopy sphere. The homotopy sphere pair $\left(M^{n}(\sigma), F_{1}^{n-2}\right)$ is called the $k$ twist-spin of $\left(M^{n-1}, N^{n-3}\right)$. Since $F_{1}^{n-2}$ carries a semifree $S^{1}$ action with codimension 2 fixed point set, $F_{1}^{n-2}$ is diffeomorphic to $S^{n-2}[\mathbf{H}]$ provided $n \geq 7$. It is an easy exercise to see that if $M^{n-1}$ is diffeomorphic to $S^{n-1}$ then $M(\sigma)$ is diffeomorphic to $S^{n}$.

The classification theorems of $\S 3$ point to an obvious generalization of this procedure. Given the knot $N^{n-3} \subset M^{n-1}(n \geq 4)$ let $k \geq 2$ and $m \geq 1$ be relatively prime integers. We have (using the same notation as in the last paragraph) $\tau=\left(M^{n-1} ; D^{n-3}, N^{n-3}-\right.$ Int $\left.D^{n-3}\right) \in \mathcal{S}_{k, m}^{n}$. The $(k, m)$ twist-spin of $(M, N)$ is the knot $F_{m}^{n-2}$ in the homotopy sphere $M(\tau)$. The $(k, m)$ and $(m, k)$ twist-spins of $(M, N)$ form a pair of knotted $n-2$ spheres in $M(\tau)$ which meet transversely in the $n-4$ sphere $F$. Of course $(k, 1)$ twist-spinning is just $k$ twist-spinning and in fact we have seen in $\$ 3$ that the result of $(k, m)$ twist-spinning may be obtained by constructing an $m$-fold branched cover over a $k$ twist-spun knot.

For a closed manifold $X$, let $X_{0}$ be the manifold obtained by removing a point from $X$. Zeeman's main theorem on twist-spun knots [Z] states that the complement of the $k$ twist-spin of $\left(M^{n-1}, N^{n-3}\right)$ fibers over the circle with fiber $V_{0}$ where $V$ is the $k$-fold cyclic branched cover of $M$ branched over $N$. More specifically, it says that the complement of the twist-spun knot is $V_{0} \times_{\phi} S^{1}$, the mapping torus of the canonical deck transformation of $V$ which rotates the normal disk of $N^{n-3}$ through the angle $2 \pi / k$.

Our generalization to $(k, m)$ twist-spinning was motivated by a question of Zeeman [Z, p. 493] as to whether it is possible to introduce a new factor into the twist-spinning process so that the complement of the knot thus obtained is $V_{0} \times_{\phi^{m}} S^{1}$ for $1<m<k$. The next theorem shows that this is exactly what we have done and also gives a streamlined proof of Zeeman's main theorem. 
THEOREM 4.1. Let $\left(M(\sigma), F_{m}\right)$ be the $(k, m)$ twist-spin of the knot $\left(M^{n-1}, N^{n-3}\right)(n \geq 4)$, and let $\mathcal{V}$ be the $k$-fold cyclic branched cover of $\left(M^{n-1}, N^{n-3}\right)$ with canonical deck transformation $\phi$. Then $M(\sigma)-F_{m}$ is diffeomorphic to $V_{0} \times_{\phi^{m}} S^{1}$.

Proof. $S^{1}$ viewed as $R / k Z$ acts on $V \times_{\phi^{m}} S^{1}$ by $s \cdot\langle y, t\rangle=\langle y, t+s\rangle$. If we view the homotopy sphere $N^{n-3}$ as the branch set of $\mathcal{V}^{n-1} \rightarrow M^{n-1}$ then for this action $E_{k}=N^{n-3} \times_{\phi^{m}} S^{1}$ and all the other orbits are principal. The orbit space is $V / \phi^{m}=V / \phi=M^{n-1}$, and $E_{k}^{*}=N^{n-3}$.

Let $x \in E_{k}$. We may identify a tube about the orbit of $x$ with $D^{n-3} \times D^{2} \times S^{1} \subset S^{n}=\partial\left(D^{n-3} \times D^{2} \times D^{2}\right)$ with the standard linear $S^{1}$ action $t \cdot(y, z, w)=\left(y, z t^{m}, s t^{k}\right)$. Perform equivariant surgery on the orbit of $x$ by replacing this tube with its complementary $\partial\left(D^{n-3} \times D^{2}\right) \times$ $D^{2}$ in $S^{n}$, and let $W^{n}$ be the resulting $S^{1}$-manifold. The result of this surgery on the orbit space $M^{n-1}$ is to remove a $D^{n-3} \times D^{2}$ whose intersection with $E_{k}^{*}$ is $D^{n-3} \times 0$ and replace it with its complementary $\partial D^{n-3} \times D^{2} \subset S^{n-1}=S^{n} / S^{1}$. Since the gluing map extends over an $n-1$ disk, the orbit space of $W^{n}$ is still $M^{n-1}$; and it is easily seen that $E^{*} \cup F^{*}=N^{n-3}$ where $F_{m}^{*}$ is an $n-3$ disk contained in $D^{n-3} \times 0 \subset$ $N^{n-3}$. Thus the structured orbit space of $W$ is in $\Im_{k, m}^{n}$ and $\left(W^{*}, E^{*} \cup F^{*}\right)$ $=(M, N)$. So it follows from Corollary 3.3 that $W^{n}$ is a homotopy sphere, and the $\operatorname{knot}\left(W^{n}, F_{m}\right)$ is the $(k, m)$ twist spin of $(M, N)$. Furthermore, $F_{m}$ is the cocore of the surgery on $V \times_{\phi^{m}} S^{1}$ by which $W$ was constructed. Thus $W-F_{m}=V_{0} \times_{\phi^{m}} S^{1}$.

Restricting the $S^{1}$ action to $Z_{k}$ or $Z_{m}$ actions we obtain counterexamples to the $n$-dimensional Smith conjecture whenever $F_{k}$ or $F_{m}$ is actually knotted. For $m= \pm 1(\bmod k)$ the examples of Giffen [Gi] and Gordon [Go] are obtained.

\section{REFERENCES}

[B] G. E. Bredon, Introduction to Compact Transformation Groups, Academic Press, New York, 1972.

[CF] P. E. Conner and E. E. Floyd, Orbit spaces of circle groups of transformations, Ann. of Math., 67 (1958), 90-98.

[DK] A. Durfee and L. Kauffman, Periodicity of branched cyclic covers, Math. Ann., 218 (1975), 157-174.

[F] R. Fintushel, Locally smooth circle actions on homotopy 4-spheres, Duke Math. J., 43 (1976), 63-70.

[Gi] C. H. Giffin, The generalized Smith conjecture, Amer. J. Math., 88 (1966), 1987-1989.

[Go] C. McA. Gordon, On the higher dimensional Smith conjecture, Proc. London Math. Soc., 29 (1974), 98-110. 
[H] W.-Y. Hsiang, On the unknottedness of the fixed point set of differentiable circle group actions on spheres - P. A. Smith conjecture, Bull. Amer. Math. Soc., 70 (1964), 687-690.

[L] J. Levine, Semi-free circle actions on spheres, Invent. Math., 22 (1973), 161-186.

[MS] J. W. Milnor and J. D. Stasheff, Characteristic Classes, Princeton University Press, 1974.

[M] P. S. Mostert (Editor), Proceedings of the Conference on Transformation Groups, New Orleans, 1967, Springer-Verlag, 1968.

[P] P. S. Pao, Nonlinear circle actions on the 4-sphere and twisting spun knots, Topology, 17 (1978), 291-296.

[S] R. Schultz, Exotic spheres admitting circle actions with codimension four stationary sets, preprint.

[St] E. V. Stein, On the orbit types in a circle action, Proc. Amer. Math. Soc., 66 (1977), $143-147$.

[Z] E. C. Zeeman, Twisting spun knots, Trans. Amer. Math. Soc., 115 (1965), 471-495.

Received June 22, 1979 and in revised form January 28, 1983. Research partially supported by grants from the National Science Foundation.

TULANE UNIVERSITY

NEW ORLEANS, LA 70118

AND

Hughes Aircraft Corporation

Los ANGEles, CA 


\title{
PACIFIC JOURNAL OF MATHEMATICS EDITORS
}

\author{
Donald BabBitT (Managing Editor) \\ University of California \\ Los Angeles, CA 90024 \\ Hugo RossI \\ University of Utah \\ Salt Lake City, UT 84112 \\ C. C. Moore and Arthur Ogus \\ University of California \\ Berkeley, CA 94720
}

J. DugundiI

Department of Mathematics

University of Southern California

Los Angeles, CA 90089-1113

R. FinN and H. SAMELSON

Stanford University

Stanford, CA 94305

\section{ASSOCIATE EDITORS}
R. ARENS
E. F. BECKENBACH
B. H. NeUmanN
F. WOLF
K. YosHida
(1906-1982)

\section{SUPPORTING INSTITUTIONS}

\author{
UNIVERSITY OF ARIZONA \\ UNIVERSITY OF BRITISH COLUMBIA \\ CALIFORNIA INSTITUTE OF TECHNOLOGY \\ UNIVERSITY OF CALIFORNIA \\ MONTANA STATE UNIVERSITY \\ UNIVERSITY OF NEVADA, RENO \\ NEW MEXICO STATE UNIVERSITY \\ OREGON STATE UNIVERSITY
}

\author{
UNIVERSITY OF OREGON \\ UNIVERSITY OF SOUTHERN CALIFORNIA \\ STANFORD UNIVERSITY \\ UNIVERSITY OF HAWAII \\ UNIVERSITY OF TOKYO \\ UNIVERSITY OF UTAH \\ WASHINGTON STATE UNIVERSITY \\ UNIVERSITY OF WASHINGTON
}

The Supporting Institutions listed above contribute to the cost of publication of this Journal, but they are not owners or publishers and have no responsibility for its content or policies.

Mathematical papers intended for publication in the Pacific Journal of Mathematics should be in typed form or offset-reproduced (not dittoed), double spaced with large margins. Please do not use built up fractions in the text of the manuscript. However, you may use them in the displayed equations. Underline Greek letters in red, German in green, and script in blue. The first paragraph must be capable of being used separately as a synopsis of the entire paper. In particular it should contain no bibliographic references. Please propose a heading for the odd numbered pages of less than 35 characters. Manuscripts, in triplicate, may be sent to any one of the editors. Please classify according to the scheme of Math. Reviews, Index to Vol. 39. Supply name and address of author to whom proofs should be sent. All other communications should be addressed to the managing editor, or Elaine Barth, University of California, Los Angeles, California 90024.

There are page-charges associated with articles appearing in the Pacific Journal of Mathematics. These charges are expected to be paid by the author's University, Government Agency or Company. If the author or authors do not have access to such Institutional support these charges are waived. Single authors will receive 50 free reprints; joint authors will receive a total of 100 free reprints. Additional copies may be obtained at cost in multiples of 50 .

The Pacific Journal of Mathematics is issued monthly as of January 1966. Regular subscription rate: $\$ 132.00$ a year (6 Vol., 12 issues). Special rate: $\$ 66.00$ a year to individual members of supporting institutions.

Subscriptions, orders for numbers issued in the last three calendar years, and changes of address should be sent to Pacific Journal of Mathematics, P.O. Box 969, Carmel Valley, CA 93924, U.S.A. Old back numbers obtainable from Kraus Periodicals Co., Route 100, Millwood, NY 10546.

The Pacific Journal of Mathematics ISSN $0030-8730$ is published monthly by the Pacific Journal of Mathematics at P.O. Box 969, Carmel Valley, CA 93924. Application to mail at Second-class postage rates is pending at Carmel Valley, California, and additional mailing offices. Postmaster: Send address changes to Pacific Journal of Mathematics, P. O. Box 969, Carmel Valley, CA 93924.

PUBLISHED BY PACIFIC JOURNAL OF MATHEMATICS, A NON-PROFIT CORPORATION

Copyright $\odot 1983$ by Pacific Journal of Mathematics 


\section{Pacific Journal of Mathematics}

Vol. 109, No. $2 \quad$ June, 1983

Tibor Bisztriczky, On the singularities of almost-simple plane curves . . . . 257

Peter B. Borwein, On Sylvester's problem and Haar spaces .............. 275

Emilio Bujalance, Cyclic groups of automorphisms of compact nonorientable Klein surfaces without boundary ............... 279

Robert Jay Daverman and John J. Walsh, Acyclic decompositions of

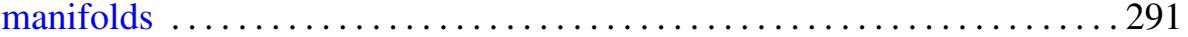

Lester Eli Dubins, Bernstein-like polynomial approximation in higher

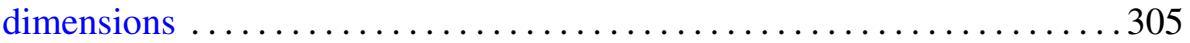

Allan L. Edelson and Jerry Dee Schuur, Nonoscillatory solutions of

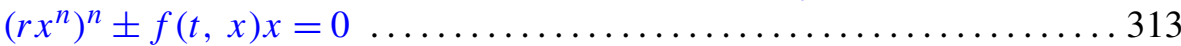

Akira Endô, On units of pure quartic number fields ................. 327

Hector O. Fattorini, A note on fractional derivatives of semigroups and

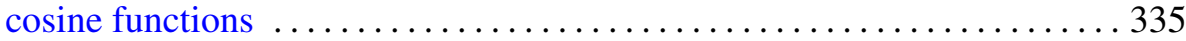

Ronald Fintushel and Peter Sie Pao, Circle actions on homotopy spheres with codimension 4 fixed point set ........................ 349

Stephen Michael Gagola, Jr., Characters vanishing on all but two

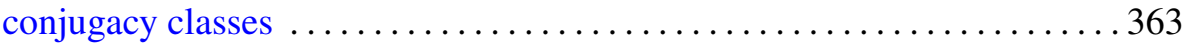

Saverio Giulini, Singular characters and their $L^{p}$ norms on classical Lie

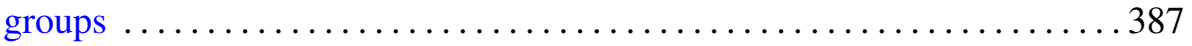

Willy Govaerts, Locally convex spaces of non-Archimedean valued continuous functions

Wu-Chung Hsiang and Bjørn Jahren, A remark on the isotopy classes of diffeomorphisms of lens spaces $\ldots . \ldots \ldots \ldots \ldots \ldots$

Hae Soo Oh, Compact connected Lie groups acting on simply connected 4-manifolds

Frank Okoh and Frank A. Zorzitto, Subsystems of the polynomial system

Knut Øyma, An interpolation theorem for $H_{E}^{\infty}$

Nikolaos S. Papageorgiou, Nonsmooth analysis on partially ordered vector spaces. II. Nonconvex case, Clarke's theory ........ 